\title{
Inter subfamily comparison of gut microbial diversity in twelve wild spider species of family Araneidae
}

\author{
Kaomud Tyagi ${ }^{1}$, Inderjeet Tyagi ${ }^{1}$, Priya Prasad $^{2}$, Kailash Chandra ${ }^{1}$, and Vikas Kumar ${ }^{1}$ \\ ${ }^{1}$ Zoological Survey of India \\ ${ }^{2}$ Affiliation not available
}

August 12, 2020

\begin{abstract}
Spiders are among the most diverse groups of arthropods remarkably known for extra oral digestion. The largest effort based on targeted 16S amplicon next generation sequencing was carried out to decipher the inter subfamily comparison of gut bacterial diversity in spiders and their functional relationship. Twelve spider species belonging to three subfamilies, Araneinae (8), Argiopinae (2) and Gasteracanthinae (2) of family Araneidae have been studied. Analysis revealed the presence of 22 phyla, 145 families, and 364 genera of microbes in the gut microbiome, with Proteobacteria as the highest abundant Phylum. Moreover, the phyla Firmicutes, Actinobacteria and Deinococcus_Thermus were also detected. The bacterial phyla Bacteriodetes and Chlamydiae dominated in Cyclosa mulmeinensis and Neoscona bengalensis respectively. At genera level, Acinetobacter, Pseudomonas, Cutibacterium, Staphylococcus, and Bacillus were the most dominant genera in their gut. In addition to this, the genus Prevotella was observed only in one species, Cyclosa mulmeinensis, and endosymbiont genus Wolbachia generally responsible for reproductive alterations was observed in one spider species Eriovixia laglaizei. Our study revealed that the gut bacterial diversity of the spiders collected from wild are quite different from the diet driven spider gut bacterial diversity as published earlier. A functional analysis revealed the involvement of gut microbiota in carbohydrate, lipid, amino acids, fatty acids and energy metabolism.
\end{abstract}

\section{Introduction}

Spiders (order Araneae) are arthropods that usually act as natural predators on insect pests in agricultural ecosystem (Michalko et al., 2018; Yang et al., 2017) bio-control agent for various diseases (Ndava et al., 2018), and indicator species for environment monitoring (Ossamy et al, 2016). They are equally important for their economical, ecological and medicinal purposes (Brinker et al., 2019). The feeding behaviour of spiders is different from that in most other arthropods, extracting nutrients by extra oral digestion (EOD). Spiders generally immobilize their prey by injecting venom and regurgitating digestive fluid onto (or into) their prey and then sucking back again the resulting liquefied tissue (Kennedy et al., 2020). This type of feeding behaviour also makes spiders an interesting model to study the composition and function of their gut microbial communities. The habitats of spiders are most diverse, i.e. desert to rain forests and it was very important to interpret the role of gut bacterial diversity of different inhabiting spiders in order to establish the host and habitat relationships (Dillon et al., 2002; Koch et al., 2011). However, the gut bacterial diversity of this most diverse group is poorly explored.

Till date, gut bacterial diversity of only six spider species (Pardosa laura, Pardosa astrigera, Nurscia albofasciata , Rabidosa rabida , Badumna longinqua, andArgiope bruennichi are known (Kennedy et al., 2020; Hu et al., 2019; Rivera et al., 2017; Sheffer et al., 2020). Kennedy et al., studied the impact of different food on the gut microbiome of the spider species Badumna longinqua, and observed that the gut bacterial diversity of this spider is generally diet-driven. In addition to this, they observed a clear correlation between the prey insects and the gut microbiome (Kennedy et al., 2020). Earlier gut microbial 
studies of three spider species observed that the phylum Proterobacteria was the most dominant, including Tenericutes, Actinobacteria, Firmicutes, Acidobacteria and Bacteroidetes. Thus, the gut bacteria of the spiders may be different from other arthropods due to their peculiar feeding style (HU et al., 2019). Rivera et al., 2017 studied the microbiome associated with the spider species Rabidosa rabida, by analysing body swaps and excreta samples. The results obtained clearly revealed the interaction between the microbes and their hosts i.e. in the presence of antimicrobial fluid, microbes like Staphylococcus aureus in body swaps and Staphylococcus sp. , in excreta samples was observed. Sheffer et al., 2020 reported the presence of novel bacterial symbiont affiliated to Tenericutes in the wasp spider i.e.Argiope bruennichi, which they named as DUSA (Dominant unknown symbiont of Argiope bruennichi ) (Sheffer et al., 2020).

In the present study, a large effort through Next Gen Sequencing (NGS) has been made to explore the gut microbiome and the predicted functional metabolism in 12 species from three spider subfamilies (Araneinae, Argiopinae and Gasteracanthinae) of the family Araneidae (orb-web weavers).

\section{Materials and Methods}

\section{Sample collection}

In this study, we have collected specimens of all the spider species from Kolkata, West Bengal State of India, except one species, Cyclosa bianchoria, which was collected from Andhra Pradesh state. The specimen were collected from the field by the following collection methods: hand picking, sweep net, and yellow pan trap. All the specimens are sorted and kept individually in a separate empty vial. After 8 hours of starvation, each specimen are transferred in $100 \%$ alcohol and stored in $4^{\circ} \mathrm{C}$. Subsequently, each specimen were washed thrice with PBS solution to remove the contamination. After washing, ten specimens of each species were pooled for DNA isolation. The spiders used in this study were non-endangered and non-protected species (Table 1). The taxonomic identification of these specimens was done by Priya Prasad on a NIKON SMZ25 stereo microscope using available keys (Tikader et al., 1982).

\section{DNA Isolation, amplification and sequencing}

The total DNA of each pooled sample of spider species was extracted using a DNeasy Blood \& Tissue Kit (Qiagen) following the manufacturer's protocol. The quantification of the DNA was checked by Qubit 2.0 Fluorometer (Q32866, Thermofisher), and the quality was checked using agarose gel electrophoresis (Cell BioScience Alphalmager MINI). The extracted DNA was amplified using the primer sets of V3V4 hypervariable regions of the 16S rRNA 341F (5'- ACTCCTACGGGAGGCAGCAG-3') and 806R (5'GGACTACHVGGGTWTCTAAT-3'). Total $25 \mu \mathrm{L}$ of mixture was prepared for the PCR, including $1 \mu \mathrm{l}$ of each primer, $0.5 \mu \mathrm{l}$ of Taq DNA polymerase (Takara), $1 \mu \mathrm{l}$ of dNTPs, $2.5 \mu \mathrm{l}$ of $10 \times$ buffer, $50 \mathrm{ng}$ of template, and Milli-Q water. The PCR cycle involved denaturation for $5 \mathrm{~min}$ at $98^{\circ} \mathrm{C}$ followed by 35 cycles for $30 \mathrm{~s}$ at $98^{\circ} \mathrm{C}$, annealing for $45 \mathrm{~s}$ at $53^{\circ} \mathrm{C}$, and elongation at $72^{\circ} \mathrm{C}$ for $45 \mathrm{~s}$, and final extension of $7 \mathrm{~min}$ at $72^{\circ} \mathrm{C}$. The PCR products were visualized using agarose gels for high-throughput sequencing of microbial diversity. The sequencing of the targeted gene region of $16 \mathrm{~S}$ rRNA was carried out on the Illumina HiSeq platform. The qualified constructed Nextra library was sequenced using PE300bp (Illumina Hiseq2500 RP V2 Kit) with rapid mode using the Hiseq 2500-RP PE301+8+8+301-v2 sequencing method. The National Center for Biotechnology Information (NCBI) GenBank Portal has been used for the submission of the generated raw reads to under the BioProject ID PRJNA638522.

\section{Bioinformatics and statistical analyses}

The generated paired end raw reads of twelve spider species were merged into single reads in QIIME2 (ver. 2019.10) (Bolyen et al., 2019) using demultiplexing. These reads were processed by DADA2 (Callahan et al., 2016) pipeline in QIIME2 for quality filtering, trimming, de-noising and merging. The chimeric reads were filtered and the non-chimeric reads were assigned into operational taxonomic units (OTUs). These OTUs were further classified based on SILVA 99\% similarity database (version 132) using QIIME2 q2-featureclassifier plugin. The generated taxonomy and feature tables along with metadata file were processed for the downstream analysis. A web-based tool, MicrobiomeAnalyst (Dhariwal et al., 2017) using Marker Data 
Profiling (MDP) module was used for bacterial diversity analysis. A total of 1326 OTUs were recovered out of 5967 OTUs after removing the singletons. These OTUs were further filtered based on low abundance features with prevalence 10 and low variance features with a default inter quantile range and resulting a total of 1175 OTUs. Further, we have also rarefy the data in normalization step with Total Sum Scaling (TSS) option.

The Alpha-diversity was analysed with T-test/ANOVA statistical methods using observed, Chao1, Shannon and Simpson as diversity measures. The PERMANOVA based statistical method for Bray Curtis and Ward's linkage based method for unweighted UniFrac distance measure were used for analyzing the beta diversity. Further, an online tool jvenn (Bardou et al., 2014) (http://jvenn.toulouse.inra.fr) was used for the construction of the Venn diagram, while the R-based (R core team 2020), Metacoder software (Foster et al., 2017) was used for the picturing of the heat tree. The $\mathbf{P}$ hylogenetic $\mathbf{I}$ nvestigation of $\mathbf{C}$ ommunities by $\mathbf{R}$ econstruction of $\mathbf{U}$ nobserved $\mathbf{S}$ tates (Langille et al., 2018) (PICRUSt2) was used for predicting the functional metabolic pathways. The predicted metabolic pathways were characterized through $\mathbf{K}$ yoto $\mathbf{E}$ ncyclopedia of $\mathbf{G}$ enes and $\mathbf{G}$ enomes (KEGG) (Kanehisa et al, 2000; Kanehisa et al., 2019; Kanehisa, $2019)^{\mathbf{2 0 - 2 2}}$ database and accuracy evaluated by the Nearest Sequenced Taxon Index (NSTI) values. The generated file along with the metadata was loaded to the STAMP (Parks et al., 2014) software for the further visualization.

\section{Results}

\section{Bacterial community composition and richness}

A total of 1077166 sequences of $16 \mathrm{~S}$ rRNA (V3-V4) were identified after pre-processing steps i.e. merging, quality filtering, chimera removal, etc. which ranges from 69573 to 106136 (average of 89763 reads per sample) and assigned into 5967 OTUs. A total of 1175 OTUs were left for downstream analysis after the removal of singletons (4641 OTUs), low variance (20 OTUs) and low abundance (131 OTUs) features. The Venn analysis revealed that 3891 OTUs were unique for Araneinae, 474 for Argiopinae and 678 for Gasteracanthinae, while 273 OTUs were shared by all the subfamilies of family Araneidae. On the other hand, the subfamily Araneinae shared 341 OTUs with Argiopinae and 275 with Gasteracanthinae. A total of 35 OTUs were shared by two subfamilies, Argiopinae and Gasteracanthinae (Figure 1a).

Taxonomic classification of microbial diversity in three subfamilies of the family Araneidae revealed the presence of total 22 phyla and 145 families. The phylum Proteobacteria was dominated in the current dataset with relative abundance of $49 \%$ for Argiopinae, $55 \%$ in Araneinae, and $75 \%$ in Gasteracanthinae. The second

dominant phylum, Firmicutes with an abundances range from 14 to $35 \%$, was detected in the guts of all the spider species. Other bacterial taxa like Actinobacteria (abundance ranges from 1 to $14 \%$ ), Bacteroidetes (1 to $10 \%$ ), and Deinococcus_Thermus (2 to $6 \%$ ) were detected in the gut of eleven spider species of the family Araneidae. The major contribution of the phylum Actinobacteria comes from two species of the subfamily Araneinae, i.e. Cyclosa spirifera and Eriovixia laglazei, while the phylum Bacteriodetes was most strongly represented in Cyclosa mulmeinensis (Araneinae). The microbiome of the species Araneus mitificus was predominantly constituted of only two phyla i.e. Proteobacteria and Firmicutes. In addition to this, the phylum Chlamydiae was observed only in one species of the subfamily Araneinae, i.e. Neoscona bengalensis (Figure 1b)

A total of 81 orders were detected in the current dataset, among them the orders that majorly contribute to the total bacterial diversity in subfamily Araneinae were Pseudomonadales andEnterobacteriales (Class: Gammaproterobacteria, Phylum: Proteobacteria), Bacilliales and Lactobacilliales (Class:Bacilli, Phylum: Firmicutes), Micrococcales, Corynebacteriales and Propionibacteriales (Class:Actinobacteria, Phylum: Actinobacteria),Flavobacteriales and Bacteriodales (Class:Bacteroidea, Phylum: Bacteriodetes), Rickettsiales (Class:Alphaproteobacteria, Phylum: Proteobacteria), Thermales (Class: Deinococci, Phylum: Deinococcus_Thermus) (Figure 2a).

Similar trends were observed in subfamily Argiopinae with considerable changes in the abundance of order Bacilliales (Class:Bacilli), Bacteriodales (Class: Bacteroidea) andRickettsiales (Class: Alphaproteobacteria 
) (Figure 2b).

The pattern of order abundance in case of subfamily Gasteracanthinae was also quite similar to subfamily Araneinae. Few considerable differences in order abdundance were observed in Enterobacteriales (Class:Gammaproterobacteria , Phylum: Proteobacteria),Lactobacilliales (Class: Bacilli, Phylum: Firmicutes),Siphingomonadales (Class: Alphaproteobacteria, Phylum: Proteobacteria), Thermales (Class: Deinococci, Phylum: Deinococcus_Thermus), Bacteriodales (Class: Bacteroidea,Phylum: Bacteriodetes) (Figure 2c).

At the family level, Moraxellaceae (20\%), Enterobacteriaceae (13\%), Bacillaceae (9\%), Pseudomonadaceae (6\%), Burkholderiaceae (5\%), were observed in all three subfamilies of spiders. The remaining $47 \%$ diversity was contributed by other families, including Prevotellaceae, Staphylococcaceae, Propionibacteriaceae, Corynebacteriaceae, Micrococcaceae, Thermaceae etc. Two families, Burkholderiaceae and Pseudomonadaceae, were not observed in one species of the subfamily Araneinae, Araneus mitificus . The major contribution of the family Prevotellaceae was reflected in only one species of the subfamily Araneinae, Cyclosa mulmeinensis (Figure 1b)

At the genus level, 364 genera were observed in the current dataset of spiders. The genera Acinetobacter , Pseudomonas, Cutibacterium, Staphylococcus, and Bacillusconstitute the major gut microbiome. In addition to this, the genusPrevotella (60\%) was observed only in one species, Cyclosa mulmeinensis while endosymbiont Wolbachia (28\%) was found only in Eriovixia laglaizei (Araneinae).

To decipher the community richness, $\alpha$ - and $\beta$-diversity analyses were carried out. The diversity measurements of Chao1, Observed, Shannon and Simpson were used for the $\alpha$-diversity analysis, while Bray-Curtis and unweighted unifrac diversity measures for $\beta$-diversity analysis. The $\alpha$-diversity for three spider subfamilies (Araneinae, Argiopinae, Gasteracanthinae) of the family Araneidae lies in the range of 3-19 (Chao1), 3-16 (Observed), 0.65-1.26 (Shannon) and 0.39-0.68 (Simpson). The changes observed in the community richness of three subfamilies were non-significant $(p>0.05)$. Moreover, the microbiome community richness for the subfamily Araneinae was higher than the community richness of Argiopinae and Gasteracanthinae (Figures 3a-d).

The unweighted unifrac based $\beta$-diversity analysis was carried out to investigate the community richness and phylogenetic arrangement of the species of three subfamilies of the family Araneidae (Figure 4). Two clades were formed and can be discriminated by the relative abundance of phylum Deinococus_Thermus. Clade I with negligible abdundance of phylum Deinococus Thermus was observed in one species of subfamily Argiopinae (Cyrtrophora cicatrosa ) + all the members of subfamily Araneinae in this clade while clade II contain all the members of subfamily Gasteracanthinae+ one species of subfamily Argiopinae (Argiope pulchella ) + two species of subfamily Araneinae (Neoscona nauticaand N. bengalensis ) (Figure 4).

Clade I was further divided into two subclades A and B: The subclade IA possesses a similar type of bacterial abundance and phylogeny except in the species Cyclosa mulmeinensis which diverged due to increased abundance of the phylum Bacteriodetes. The subclade IB also possesses a similar type of bacterial abundance and phylogeny except in the species of Cyclosa bianchoria, in which the abundance of phylum Proteobacteria was higher than the other three species. Two species of Araneinae (Eriovixia excelsa and Cyclosa spirifera) and one species of Argiopinae (Cyrtophora cicatrosa) in subclade IB have similar type of bacterial diversity and phylogeny, whileCyclosa spirifera was branched out from this subclade due to the more abundance of phylum Actinobacteria.

The clade II with a considerable abundance of Deinococcus Thermus contains five species (Neoscona nautica , $N$.bengalensis, Gasteracantha kuhli, Gasteracantha hasselti and Argiope pulchella). The branch of speciesArgiope pulchella (Argiopinae) in Clade II was the first branch out due to the high abundance of phylum Firmicutes. This clade further sub divided into two subclades A and B and have similar type of bacterial diversity and phylogeny except few changes in the diversity of Phylum Chalamydiae and Cyanobacteria.

The Bray-Curtis based NMDS ordination plot also suggested a similar type of results as indicated by un- 
weighted unifrac diversity measures. Based on distance matrix (NMDS Stress $=0.055$ ), it was observed that two species i.e. Araneus mitificus and Eriovixia laglzei were in close resemblance with each other. The gut samples obtained from the two species (Gasteracantha kuhli and G. hassleti) of subfamily Gasteracanthinae were in close resemblance with one species of Argiopinae (Argiope pulchella) and two species of Araneinae (Neoscona nautica and $N$. bengalensis ). Rest of the members of subfamily Araneinae were in close resemblance with one species of family Argiopinae (Cytrophora cicatrosa) (Figure 5).

The PICRUSt2 computational approach was used to decipher the resemblances and differences in metabolic profiles of the microbiome of three subfamilies, i.e. Araneinae, Argiopinae, Gastercanthinae. Comparative studies of metabolic functional gene abundance of these three families predict that the subfamily Araneinae shared 11 metabolic pathways with Argiopinae and 77 pathways with Gasteracanthinae, while Argiopinae shared 16 pathways with Gasteracanthinae. Seven metabolic pathways were shared among all three subfamilies, i.e. carbohydrate metabolism, amino acid biosynthesis (Arginine, Lysine, Isolucine), amino acids degradation and fatty acids metabolism (Figure 6).

The metabolic pathways of gut bacteria included between subfamily Araneinae and Gasteracanthinae were carbohydrate metabolism, fatty acids metabolism, amino acids biosynthesis and degradation, metabolism of cofactors and vitamins, energy metabolism, and nucleotide metabolism. The gut bacteria involved in amino acid metabolism and carbohydrate metabolism showed higher relative abundances than the bacteria involved in the other metabolism. Whereas, the metabolic pathways of gut bacteria included between subfamily Araneinae and Argiopinae were carbohydrate metabolism and degradation, amino acid biosynthesis.

\section{Discussion}

In the present study, the largest effort has been made to map the gut microbiome and their predicted functional pathways in metabolic activities in 12 species under three spider subfamilies i.e. Araneinae, Argiopinae and Gasteracanthinae of family Araneidae. Targeted 16S rRNA amplicon analysis reveals the presence of 22 phyla and 145 families. The dominant phyla were Proteobacteria, Firmicutes, Actinobacteria, Bacteroidetes etc. Moreover, the dominant genera in phylum Proteobacteria were Acinetobacter, Pseudomonas ,Enterobacter with considerable abundance throughout the spider species, while an endosymbiont genus Wolbachia was observed only in one spider species i.e. Eriovixia laglaizei. Subsequently, genera like Staphylococcus and Bacillus (phylum Firmicutes) and Cutibacterium (Actinobacteria) were the most abundant. In addition to this, the genus Prevotella(phylum Bacteroidetes) was most abundant in one species of spider i.e.Cyclosa mulmeinensis which may be due to digested grass grub larvae. This report is also observed in human gut microbiome which was modified due to digestion of grass grub larvae (Young et al. 2020).

The high abundance of representatives of the phylum Proteobacteria in the spiders' gut is in line with previous studies of other arthropods (Hu et al., 2019; Chen et al., 2016; Ruokolainen et al., 2016; Hammer et al., 2017). The genera, Acinetobacter and Pseudomonas were known for their role in energy, lipid metabolism and degradation of organophosphate based insecticides (Engel \& Morgan, 2013; Van Dexter \& Boopathy, 2019; Itoh et al., 2018) as reported earlier in other insects like weevils (Briones-Roblero et al., 2017), Cerambycidae (Delalibera et al., 2005), and silkmoth (Anand et al., 2010). Moreover, a few strains of the genus Pseudomonas showed active antagonism towards entomopathogenic fungi (Indiragandhi et al., 2007). Furthermore, members of the genus Enterobacter were responsible for enzyme metabolism through superoxide dismutase or catalase enzyme activity (Xia et al., 2017). The endosymbiont genus Wolbachia is known for their proliferative and transmitting activities (Bi \& Wang, 2020) by altering the sex ratio of their host reproduction (Hu et al., 2019).

The genera Staphylococcus and Bacillus may also be involved in the protease activity in the gut of spiders as reported earlier (Paniagua Voirol et al., 2018). Staphylococcus contains Gram-positive bacteria which are usually non-pathogenic, exceptStaphylococcus aureus (He et al., 2013). Furthermore, the species of the genus Bacillus were responsible for the enzyme and phospholipids metabolism (Nigris et al., 2018; Favaro et al., 2016). One species of the genus Bacillus ,B. thuringiensis, is commonly used as an insect pathogen (Joan \& Foster, 2011). 
The phylum Actinobacteria was the third most abundant taxon in the gut of spider. Members of Actinobacteria are known for producing food processing enzymes, nutrient synthesis and bioactive metabolites against the invasion of the pathogenic bacteria (Kaltenpoth, 2009). The genus Cutibacterium may be responsible for the fatty acid metabolism (Rocha Martin et al., 2019) as indicated by PICRUSt2 analysis also. In the case of the phylum Bacteroidetes, the genus Prevotella has been reported as being responsible for the histolysis in the larval stages and histogenesis in the adult developmental stages in bark beetle (Dendroctonus rhizophagus ) (Briones-Roblero et al., 2017).

The gut bacterial diversity assessment in spiders indicated, that the gut microbiota can be greatly influenced by factors like habitat, feeding behaviour and host which in turn responsible for different metabolic activities related to self-defence against natural enemies, digestion and detoxification as indicated earlier (Hu et al., 2019). In addition to this, specimens collected from the field will be of great significance in terms of actual gut bacterial diversity and their potential role in metabolism. Moreover, it can be used as baseline data in the future to distinguish the bacterial diversity present in the gut of spiders from the natural environment and that of laboratory reared specimens.

\section{Conclusion}

In our study, we mapped the bacterial diversity of 12 species of spiders using the NGS 16S rRNA (V3V4) sequence data. The genusPrevotella was observed only in Cyclosamulmeinensis, and endosymbiont Wolbachia detected for the first time in Eriovixia laglaizei. This alphaproteobacteria endosymbiont had previously been reported in another species of the genus Eriovixia, E. cavaleriei (Wang et al, 2010). It prompts for the screening of spiders for Wolbachia and its role in metabolism. The dendrogram based on the unweighted unifrac $\beta$-diversity analysis clearly indicates that the gut microbiota composition in spiders is not correlated with the phylogenetic relationship of these species, suggesting that the spider microbiota may be influenced by the feeding behavior and environmental factors like host and habitat. Our study also revealed that the field collected spider gut bacterial diversity are quite different from the diet driven spider gut bacterial diversity as published earlier. Given the high bacterial diversity in the gut of spiders as detected in the present study, a larger number of spider species needs to be mapped for their gut microbiome to get a clear picture of diversity and the role of the gut microbiome in spiders.

\section{Acknowledgement(s)}

Authors are thankful to the Director, Zoological Survey of India, Kolkata, for providing constant support and facilities to carry out the studies. We thank Prof. Rainer Breitling, University of Manchester for language editing. The study is financially supported by core funding of Zoological Survey of India, Kolkata, Ministry of Environment Forest and Climate Change.

\section{Author Contributions}

PP involved in specimen collection, identification and DNA isolation; KT and VK involved in Conceptualization, Data Curation, Methodology, Software; KT, IT and VK involved in Bioinformatics analysis, Manuscript writing and Editing. KC and VK supervised the project and Funding acquisition.

\section{Data Accessibility}

The raw reads were submitted to National Center for Biotechnology Information (NCBI) GenBank under the BioProject ID: PRJNA638522.

\section{References}

Anand, A.A.P., Vennison, S.J., Sankar, S.G., Prabhu, D.I.G., Vasan, P.T., Raghuraman T., Geoffrey, C.J., Vendan, S.E. (2010). Isolation and characterization of bacteria from the gut of Bombyx mori that degrade cellulose, xylan, pectin and starch and their impact on digestion. J Insect Sci., 10, 107.

Bardou, P., Mariette, J., Escudié, F., Djemiel, C., Klopp. C. (2014). jvenn: an interactive Venn diagram viewer. BMC Bioinf. , 15, 293-300. 
Bi, J., \& Wang, Y.F. (2020). The effect of the endosymbiont Wolbachia on the behavior of insect hosts. Insect science, $1-13$.

Bolyen, E., Rideout, J.R., Dillon, M.R., et al., (2019). Reproducible, interactive, scalable and extensible microbiome data science using QIIME 2. Nat. Biotechnol., 37, 852-857.

Brinker, P., Fontaine, M.C., Beukeboom, L.W., Salles, J.F. (2019). Host, symbionts, and the microbiome: the missing tripartite interaction. Trends Microbiol. , 27, 480-488.

Briones-Roblero C.I., RodrIguez-Diaz, R., Santiago-Cruz J.A., Zuniga, G., Rivera- Orduna, F. (2017). Degradation capacities of bacteria and yeasts isolated from the gut of Dendroctonus rhizophagus(Curculionidae: Scolytinae). Folia Microbiol. , 62, 1-9.

Briones-Roblero, C.I., Hernández-García, J.A., Gonzalez-Escobedo, R., Soto-Robles, L.V., Rivera-Orduña, F.N., Zúniga, G. (2017). Structure and dynamics of the gut bacterial microbiota of the bark beetle,Dendroctonus rhizophagus (Curculionidae: Scolytinae) across their life stages. PloS one, 12, e0175470.

Callahan, B.J., McMurdie P.J., Rosen, M.J., Han, A.W., Johnson, A.J., Holmes, S.P. (2016) DADA2: Highresolution sample inference from Illumina amplicon data, Nat. Methods, 13, 581-583.

Chen, B., Beng-Soon, T., Sun, C., Hu, S., Lu, X., Wilhelm, B., Shao, Y. (2016). Biodiversity and activity of the gut microbiota across the life history of the insect herbivore Spodoptera littoralis . Sci. Rep. , 6, 29505.

Delalibera I. Jr, Handelsman J., Raffa, K.F. (2005). Contrasts in cellulolytic activities of gut microorganisms between the wood borer,Saperda vestita (Coleoptera:Cerambycidae), and the bark beetles,Ips pini and Dendroctonus frontalis (Coleoptera: Curculionidae). Environ Entomol., 34, 541-547.

Dhariwal, A., Chong, J., Habib, S., King, I., Agellon, L.B., Xia. J. (2017). "MicrobiomeAnalyst - a webbased tool for comprehensive statistical, visual and meta-analysis of microbiome data", Nucleic Acids Res. , 45,180-188.

Dillon, R.J., Vennard, C.T., Charnley, A.K. (2002). A note: gut bacteria produce components of a locust cohesion pheromone. J. Appl. Microbiol., 92, 759-763.

Engel, P., \& Moran, N.A. (2013). The gut microbiota of insects-diversity in structure and function. FEMS Microbiol. Rev., 37, 699-735.

Favaro, G., Bogialli, S., Di Gangi, I.M., Nigris, S., Baldan, E., Squartini, A., Pastore, P., Baldan, B. (2016). Characterization of lipopeptides produced by Bacillus licheniformis using liquid chromatography with accurate tandem mass spectrometry. Rapid Commun Mass Sp , 30, 2237-2252.

Foster Z.S.L., Sharpton T.J., Grunwald N.J. (2017). Metacoder: An R package for visualization and manipulation of community taxonomic diversity data. PLoS Comput. Biol. , 13, e1005404.

Hammer, T.J., Janzen, D.H., Hallwachs, W., Jaffe, S.P., Fierer, N. (2017). Caterpillars lack a resident gut microbiome. Proc. Natl. Acad. Sci., 114, 9641-9646.

He, C., Nan, X., Zhang, Z., Li, M. (2013). Composition and diversity analysis of the gut bacterial community of the Oriental armyworm, Mythimna separata, determined by culture-independent and culture-dependent techniques. J. Insect Sci. , 13, 165.

Hu, G., Zhang, L., Yun, Y., Peng, Y. (2019). Taking insight into the gut microbiota of three spider species: No characteristic symbiont was found corresponding to the special feeding style of spiders. Ecol. Evol., 9: $8146-8156$.

Indiragandhi, P., Anandham, R., Madhaiyan, M., Poonguzhali, S., Kim, G.H., Saravanan, V.S. \& Sa, T. (2007). Cultivable bacteria associated with larval gut of prothiofos-resistant, prothiofos-susceptible and field-caught populations of diamondback moth, Plutella xylostella and their potential for, antagonism towards entomopathogenic fungi and host insect nutrition. J. Appl. Microbiol., 103, 2664-2675. 
Itoh, H., Hori, T., Sato, Y., Nagayama, A., Tago, K., Hayatsu, M., Kikuchi, Y. (2018). Infection dynamics of insecticide-degrading symbionts from soil to insects in response to insecticide spraying.ISME J. , 12, 909-920.

Joan L.S., Foster, J.W., (2011), Microbiology: An Evolving Science (2nd Edition), Norton

Kaltenpoth M. (2009). Actinobacteria as mutualists: general healthcare for insects?. Trends Microbiol. , $17,529-535$.

Kanehisa, M. \& Goto, S. (2000). KEGG: Kyoto Encyclopedia of Genes and Genomes, Nucleic Acids Res. , $28,27-30$.

Kanehisa, M., Sato, Y., Furumichi, M. Morishima, K., Tanabe, M. (2019). New approach for understanding genome variations in KEGG. Nucleic Acids Res ., 47, D590-D595.

Kanehisa, M; Toward understanding the origin and evolution of cellular organisms. Protein Sci . 28, 19471951.

Kennedy, S.R. Tsau, S., Gillespie, R., Krehenwinkel, H. (2020). Are you what you eat? A highly transient and prey-influenced gut microbiome in the grey house spider Badumna longinqua, Mol. Ecol. , 29, 1001-1015.

Koch, H., Hempel, P.S. (2011). Bacterial communities in central European bumblebees: low diversity and high specificity. Microb. Ecol.,62, 121-133.

Langille, M.G.I. (2018). Exploring Linkages between Taxonomic and Functional Profiles of the Human Microbiome, mSystems, 3, e00163-17.

Michalko, R., Pekar, S., Entling, M.H. (2018). An updated perspective on spiders as generalist predators in biological control. Oecologia,189, 21-36.

Ndava, J., Llera, S.D., Manyanga, P. (2018). The future of mosquito control: The role of spiders as biological control agents: A review.Int. J. Mosquito Res. , 5, 6-11.

Nigris, S., Baldan, E., Tondello, A., Zanella, F., Vitulo, N., Favaro, G., Guidolin, V., Bordin, N., Telatin, A., Barizza, E., Marcato, S., Zottini, M., Squartini, A., Valle, G., Baldan, B. (2018), Biocontrol traits of Bacillus licheniformis GL174, a culturable endophyte of Vitis vinifera cv. Glera". BMC Microbiol., 18, 133.

Ossamy, S., Elbanna, S.M., Orabi, G.M., Semida, F.M. (2016). Assessing the potential role of spider as bioindicators in Ashtoumel Gamil Natural Protected Area, Port Said, Egypt. Indian J. Arachnol., 5, 100112.

Paniagua Voirol, L.R., Frago, E., Kaltenpoth, M., Hilker, M., \& Fatouros, N.E. (2018). Bacterial symbionts in Lepidoptera: Their diversity, transmission, and impact on the host, Front. Microbiol., 9, 556.

Parks D.H., Tyson G.W., Hugenholtz P., Beiko R.G. (2014). STAMP: statistical analysis of taxonomic and functional profiles, Bioinformatics , 30, 3123-3124.

R Core Team. (2020). R: A language and environment for statistical computing. R Foundation for Statistical Computing, Vienna, Austria. URL http://www.R-project.org/.

Rivera, P., Stork, R., Hug, A. (2017). A First Look at the Microbial Community of Rabidosa rabida, a Wolf Spider in Searcy, Arkansas, Journal of the Arkansas Academy of Science, 71, 13.

Rocha Martin, V.N., Schwab, C., Krych, L., Voney, E., Geirnaert, A., Braegger, C., Lacroix, C. (2019). Colonization of Cutibacterium avidum during infant gut microbiota establishment. FEMS Microbiol. Ecol. , 95, 1-14.

Ruokolainen, L., Ikonen, S., Makkonen, H., Hanski, I. (2016). Larval growth rate is associated with the composition of the gut microbiota in the glanville fritillary butterfly. Oecologia , 181, 895-903. 
Sheffer, M.M., Uhl, G., Prost, S., Lueders, T., Urich, T., Bengtsson, M.M. (2020). Tissue-and PopulationLevel Microbiome Analysis of the Wasp Spider Argiope bruennichi Identified a Novel Dominant Bacterial Symbiont, Microorganisms , 8, 8.

Tikader, B.K. (1982) Part 1. Family Araneidae (Argiopidae). Typical orb-weavers. In: The fauna of India. Spiders: Araneae. Vol. II. Zoological Survey of India, Calcutta, 1-293.

Van Dexter, S., Boopathy, R. (2019). Biodegradation of phenol by Acinetobacter tandoii isolated from the gut of the termite.Environ Sci Pollut Res. 26, 34067-72.

Wang, Z.Y., Deng, C., li-Yun, Y, Jian, C., Peng, Y. (2010). Molecular detection and the phylogenetics of Wolbachia in Chinese spiders (Araneae), J Arachnol , 38, 237-241.

Xia, X., Gurr, G.M., Vasseur, L., Zheng, D., Zhong, H., Qin, B., et al., (2017). Metagenomic sequencing of diamond back moth gut microbiome unveils key holobiont adaptations for herbivory. Front. Microbiol., 8, 663.

Yang, T.B., Liu, J., Yuan, L.-yu, Zhang, Y., Li, D., Agnarsson, I., Chen. J. (2017). Molecular identification of spiders preying onEmpoasca vitis in a tea plantation." Sci. Rep., 7, 1-10.

Young, W., Arojju, S.K., McNeill, M.R., Rettedal, E., Gathercole, J., Bell, N. Payne, P. (2020). Feeding Bugs to Bugs: Edible Insects Modify the Human Gut Microbiome in an in vitro Fermentation Model. Front. Microbiol., 11, p.1763.

Table 1

List of the species studied in this study with their code.

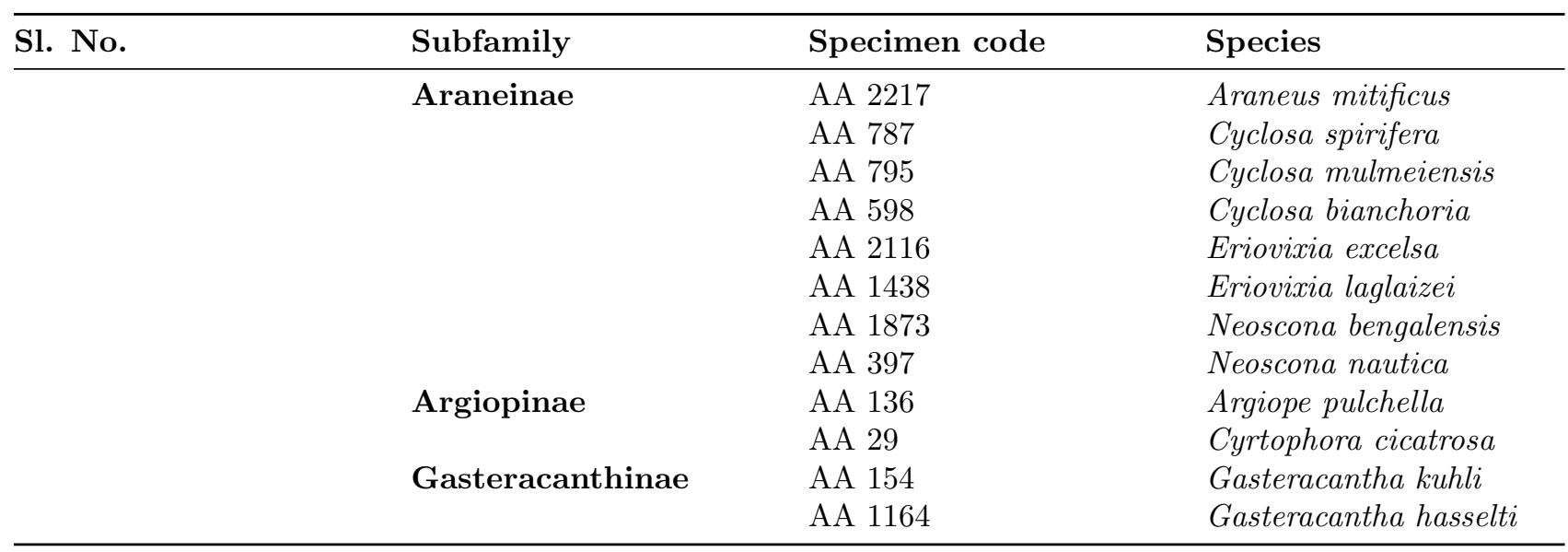

\section{Figure Captions}

Figure 1. (a) Venn diagram (b) Abundance of gut bacterial diversity at the phyla and family level of twelve spider species.

Figure 2a. Heat tree (to order level) of subfamily Araneinae to demonstrate the gut bacterial diversity in three subfamilies of the family Araneidae. The R based software (http://www.R-project.org/) Metacoder has been used to drawn these figures and further edited in Adobe Photoshop.

Figure 2b. Heat tree (to order level) of subfamily Argiopinae to demonstrate the gut bacterial diversity in three subfamilies of the family Araneidae. The R based software (http://www.R-project.org/) Metacoder has been used to drawn these figures and further edited in Adobe Photoshop.

Figure 2c. Heat tree (to order level) of subfamily Gasteracanthinae to demonstrate the gut bacterial 
diversity in three subfamilies of the family Araneidae. The R based software (http://www.R-project.org/) Metacoder has been used to drawn these figures and further edited in Adobe Photoshop.

Figure 3. Box plot for the alpha-diversity index (a) observed, (b) Chao1, (c) Shannon and (d) Simpson of the gut bacterial diversity in three subfamilies of family Araneidae. The ends of the whiskers represent the minimum and maximum while the line inside the box represents the median.

Figure 4. Dendrogram of $\beta$-diversity of twelve species from three subfamilies of the family Araneidae, along with phylum gut bacterial diversity.

Figure 5. Bray-Curtis dissimilarity-based Non-metric Multidimensional Scaling (NMDS) ordination plot of twelve spider species.

Figure 6 . Stacked column bar graph representing the predicted functional metabolic pathway comparison between three subfamilies of family Araneidae. All sequence reads were used to predict functions against the KEGG database (http://www.genome.jp/kegg/), which is implemented in PICRUSt2 (https://github.com/picrust/picrust2) bioinformatics software package. 


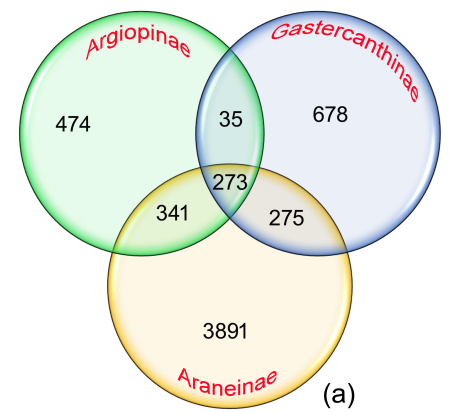

(b)
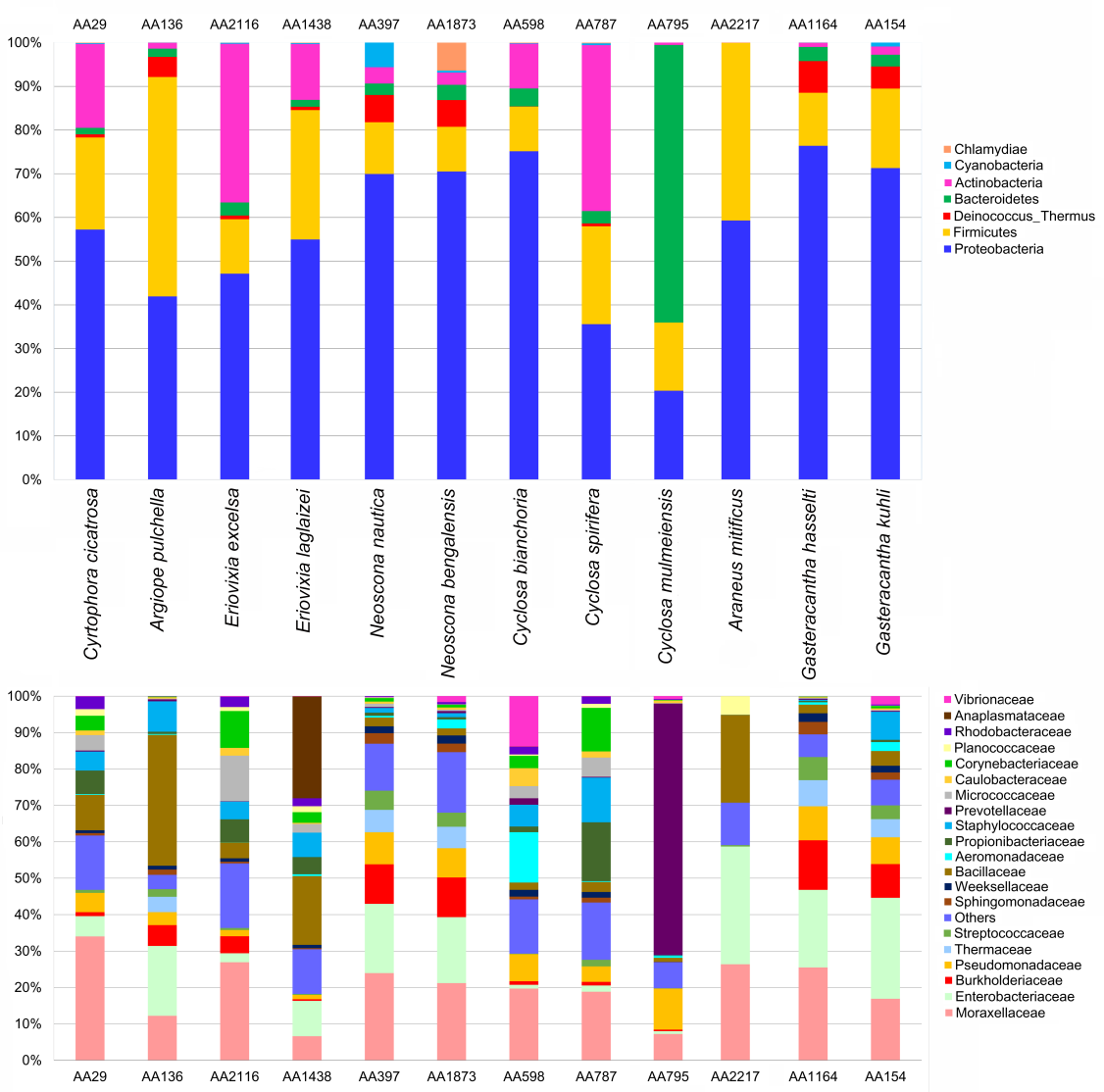


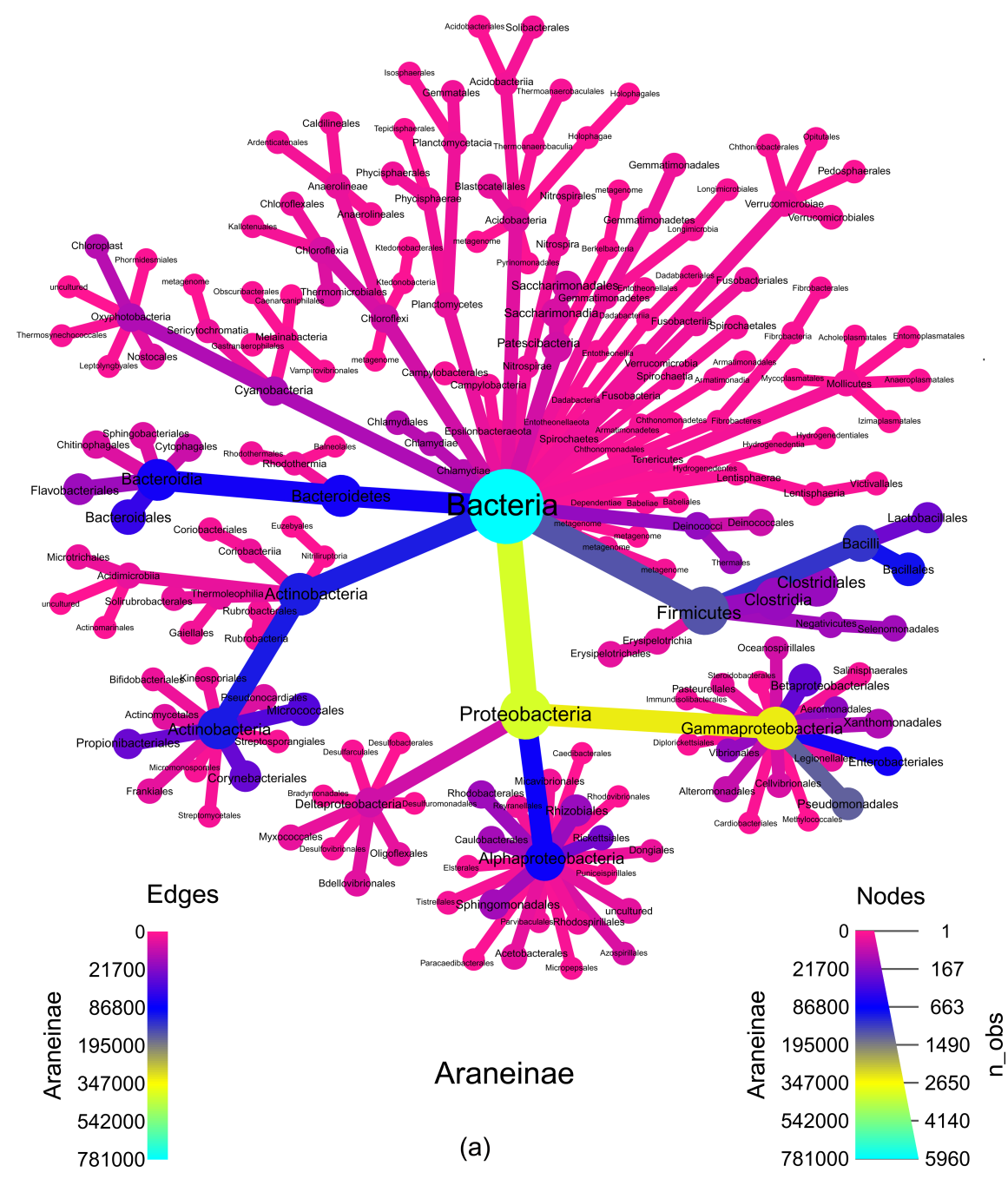




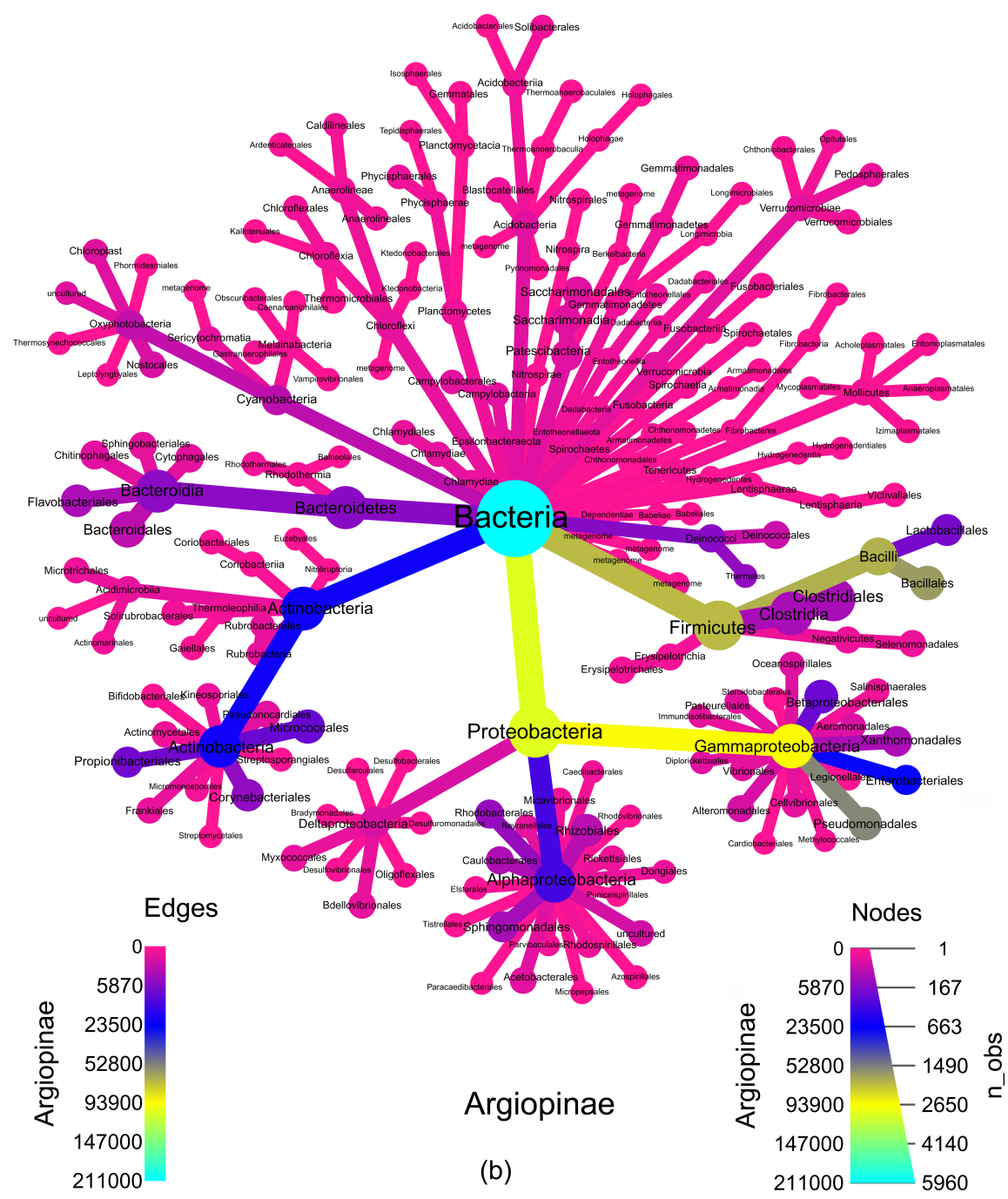




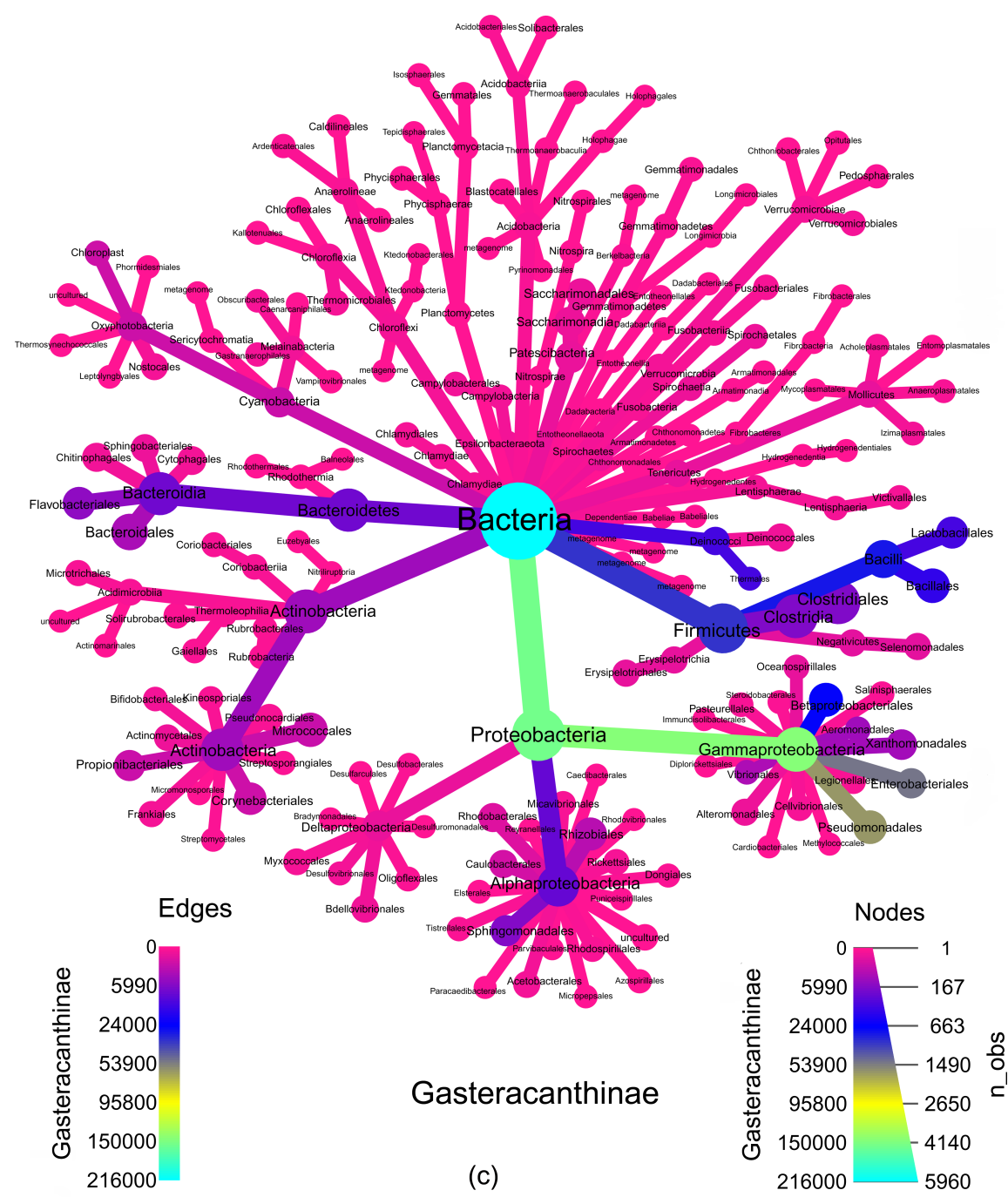


(a)
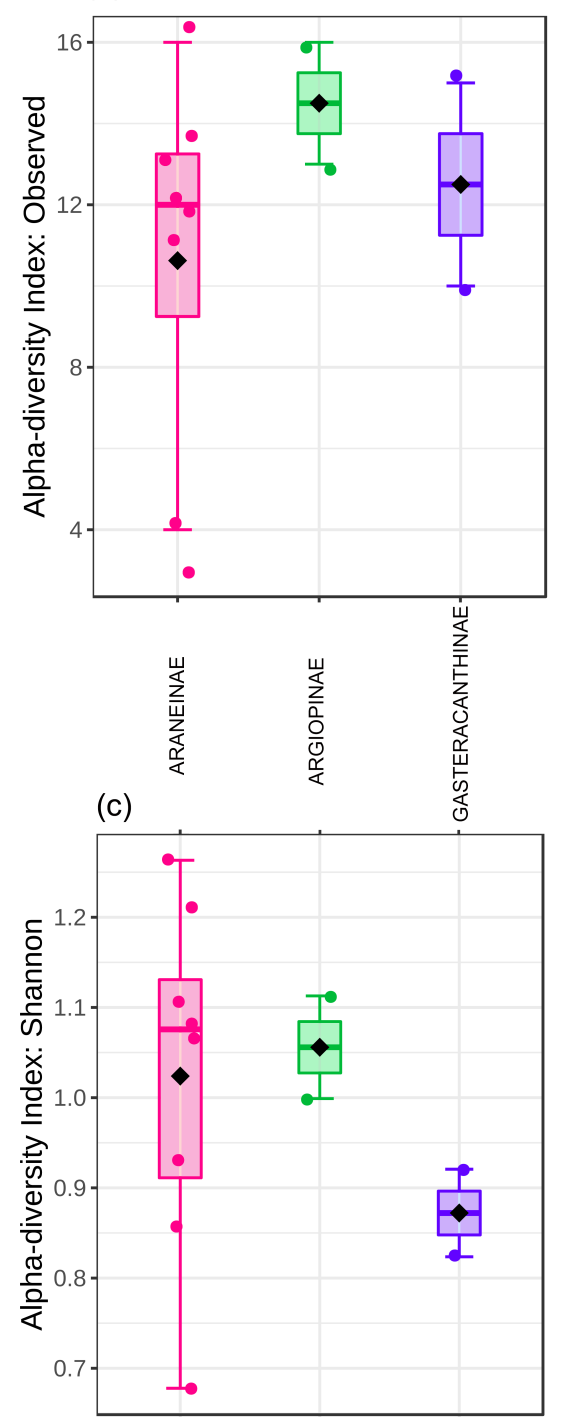

(b)
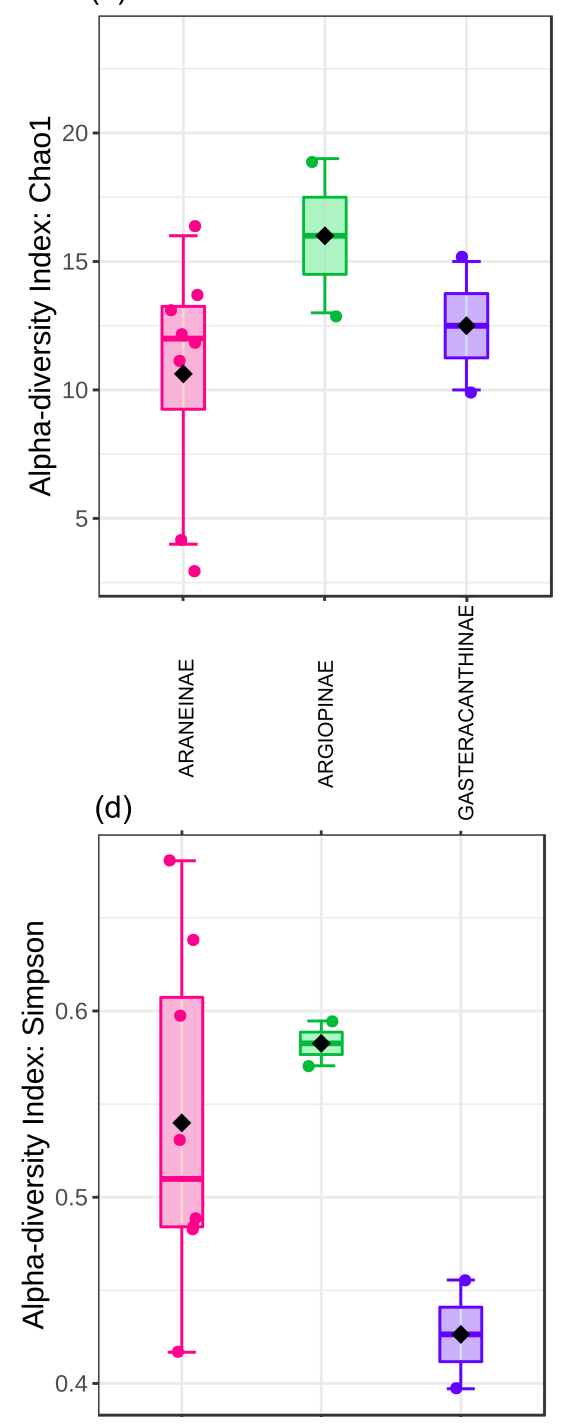


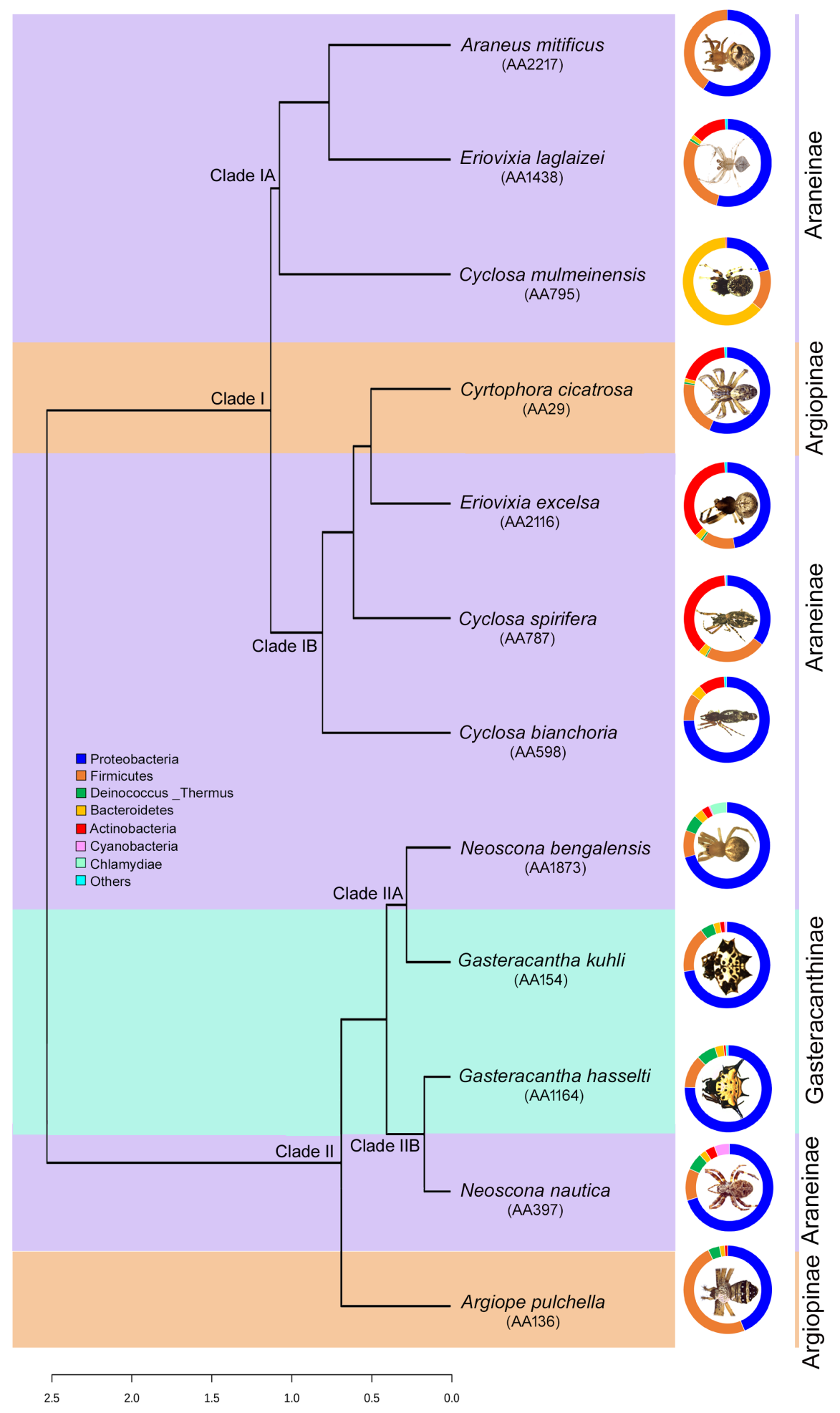




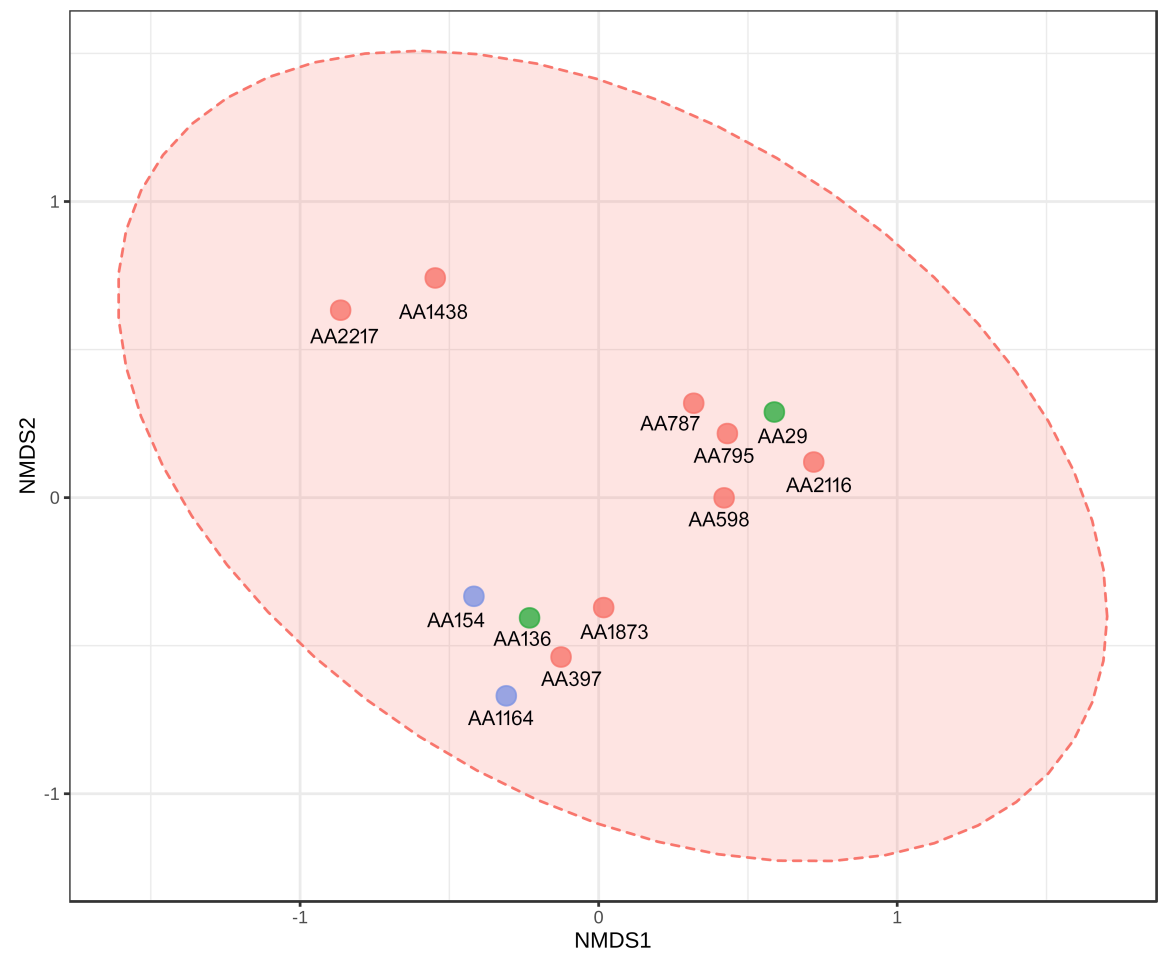


$\square$ Araneinae $\square$ Gasteracanthinae

\begin{tabular}{|c|c|}
\hline $\begin{array}{l}\text { L-lysine biosynthesis III } \\
\text { polymyxin resistance }\end{array}$ & 巴寻 \\
\hline \multirow{2}{*}{\multicolumn{2}{|c|}{$\begin{array}{r}\text { superpathway of pyridoxal 5'-phosphate biosynthesi... } \\
\text { methylphosphonate degradation I }\end{array}$}} \\
\hline & \\
\hline L-lysine biosynthesis VI E & ב \\
\hline fatty acid \&beta;-oxidation I E & \\
\hline \multicolumn{2}{|l|}{ superpathway of L-arginine and L-ornithine degrada... } \\
\hline \multicolumn{2}{|l|}{ superpathway of L-arginine, putrescine, and 4-amin... } \\
\hline D-glucarate degradation I $\mathrm{E}$ & \\
\hline \multicolumn{2}{|l|}{ nylon-6 oligomer degradation $\mid$} \\
\hline L-histidine degradation II E & ق \\
\hline \multicolumn{2}{|l|}{ superpathway of D-glucarate and D-galactarate degr... ש } \\
\hline \multicolumn{2}{|l|}{ D-galactarate degradation I ש } \\
\hline \multicolumn{2}{|l|}{$\begin{array}{l}\text { creatinine degradation I } \\
\text { superpathway of phospholipid biosynthesis I (bacte... }\end{array}$} \\
\hline superpathway of phospholipid biosynthesis I (bacte... E & \\
\hline \multicolumn{2}{|c|}{ superpathway of geranylgeranyl diphosphate biosynt... } \\
\hline glycolysis I (from glucose 6-phosphate) & 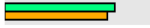 \\
\hline \multicolumn{2}{|l|}{ androstenedione degradation $\mid$} \\
\hline \multicolumn{2}{|l|}{ ethylmalonyl-CoA pathway P } \\
\hline vitamin E biosynthesis (tocopherols) | & \\
\hline \multicolumn{2}{|c|}{ UDP-N-acetylmuramoyl-pentapeptide biosynthesis II ... } \\
\hline \multicolumn{2}{|c|}{ biotin biosynthesis I } \\
\hline \multicolumn{2}{|l|}{ uperpathway of hexuronide and hexuronate degradation } \\
\hline \multicolumn{2}{|l|}{ superpathway of adenosine nucleotides de novo bios... } \\
\hline \multicolumn{2}{|c|}{ L-arginine degradation II (AST pathway) } \\
\hline \multicolumn{2}{|l|}{ superpathway of $\mathrm{C} 1$ compounds oxidation to $\mathrm{CO} 21$} \\
\hline \multicolumn{2}{|l|}{ superpathway of sulfolactate degradation | } \\
\hline phospholipases | & \\
\hline \multicolumn{2}{|l|}{ CMP-legionaminate biosynthesis II } \\
\hline \multicolumn{2}{|l|}{ glycolysis III (from glucose) E } \\
\hline ergothioneine biosynthesis I (bacteria) | & \\
\hline 8-amino-7-oxononanoate biosynthesis I & בב \\
\hline peptidoglycan biosynthesis $\mathrm{V}$ (\&beta;-lactam resist... & \\
\hline pyrimidine deoxyribonucleotide phosphorylation $E$ & E \\
\hline superpathway of ornithine degradation $\mathrm{E}$ & ב \\
\hline L-arginine biosynthesis IV (archaebacteria) $E$ & \\
\hline spirilloxanthin and 2,2'-diketo-spirilloxanthin bi... | & \\
\hline palmitate biosynthesis II (bacteria and plants) & בـ ב \\
\hline L-arginine biosynthesis I (via L-ornithine) & $\Xi$ \\
\hline superpathway of guanosine nucleotides de novo bios... $E$ & \\
\hline nicotinate degradation I & \\
\hline sucrose degradation II (sucrose synthase) P & \\
\hline mandelate degradation to acetyl-COA | & \\
\hline superpathway of purine nucleotides de novo biosynt... $E$ & $\Xi$ \\
\hline meta cleavage pathway of aromatic compounds $P$ & \\
\hline ppGpp biosynthesis $E$ & \\
\hline mandelate degradation II & \\
\hline peptidoglycan biosynthesis III (mycobacteria) $\mathrm{E}$ & Э \\
\hline 2-aminophenol degradation | & \\
\hline superpathway of adenosine nucleotides de novo bios... $E$ & $\Xi$ \\
\hline superpathway of purine nucleotides de novo biosynt... $E$ & E \\
\hline NAD salvage pathway $\| \mathrm{E}$ & ב \\
\hline peptidoglycan biosynthesis I (meso-diaminopimelate... $E$ & E \\
\hline methanol oxidation to carbon dioxide $\mathrm{F}$ & \\
\hline superpathway of guanosine nucleotides de novo bios... & קבק \\
\hline sitosterol degradation to androstenedione & \\
\hline fatty acid salv & \\
\hline ation II (meta-cle & \\
\hline ation I (serine pathway & \\
\hline
\end{tabular}

superpathway of (Kdo)2-lipid A biosynthesis enterobacterial common antigen biosynthesis 已

benzoyl-CoA degradation II (anaerobic) | superpathway of mycolyl-arabinogalactan-peptidogly... aerobactin biosynthesis 8

guanosine ribonucleotides de novo biosynthesis

catechol degradation to 2-oxopent-4-enoate II mixed acid fermentation $\rightleftharpoons$ factor 420 biosynthesis 3-hydroxypropanoate cycle superpathway of pyrimidine ribonucleotides de novo... tRNA processing

UDP-N-acetylmuramoyl-pentapeptide biosynthesis I (... 2-amino-3-carboxymuconate semialdehyde degradation... L-arginine biosynthesis II (acetyl cycle) adenosine deoxyribonucleotides de novo biosynthesi... guanosine deoxyribonucleotides de novo biosynthesi... S-adenosyl-L-methionine cycle

$0.0 \quad 0.7$
Mean proportion (\%)

\section{$\square$ Araneinae $\square$ Argiopinae}

superpathway of L-methionine biosynthesis (by sulf. L-isoleucine biosynthesis IV superpathway of mycolyl-arabinogalactan-peptidogly... adenosycobalamin salvage from cobinamide II sitosterol degradation to androstenedione L-isoleucine biosynthesis III L-arginine biosynthesis II (acetyl cycle)
benzoyl-CoA degradation II (anaerobic)

Calvin-Benson-Bassham cycle

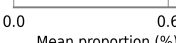

Argiopinae $\square$ Gasteracanthinae

$$
\begin{aligned}
& \text { glycolysis I (from glucose 6-phosphate) } \\
& \text { L-arginine biosynthesis II (acetyl cycle) } \\
& \text { (5Z)-dodec-5-enoate biosynthesis } \\
& \text { L-lysine biosynthesis III } \\
& \text { oleate biosynthesis IV (anaerobic) }
\end{aligned}
$$
superpathway of L-isoleucine biosynthesis I fucose degradation $b$ superpathway of L-threonine biosynthesis chlorophyllide a biosynthesis III (aerobic, light ... B chlorophyllide a biosynthesis II (anaerobic) I -isoleucine biosynthesis III taxadiene biosynthesis (engineered) mycolate biosynthesis palmitoleate biosynthesis I ffrom (57)-dodec-5 fatty acid \&beta;-oxidation methylphosphonate degradation I 